Pacific Journal of Mathematics

FINITE GROUP ACTION AND EQUIVARIANT BORDISM 


\title{
FINITE GROUP ACTION AND EQUIVARIANT BORDISM
}

\author{
S. S. KHARE
}

\begin{abstract}
Conner and Floyd proved that if $\mathrm{Z}_{2}^{k}$ acts on a closed manifold $M$ differentiably and without any fixed point, then $M$ is a boundary. Stong gave a stronger result proving that if $(M, \theta)$ is a closed $\mathrm{Z}_{2}^{k}$-differential manifold with no stationary point, then $(M, \theta)$ is a $\mathbf{Z}_{2}^{k}$-boundary. In the present note, we discuss this problem for a finite group in detail. Let $G$ be a finite group. By the 2-central component $G_{2}(C)$ of $G$, we will mean the subgroup of $G$ consisting of the identity element and all the elements of order 2 in the center of $G$. We prove in this note that the fixed data of the 2-central component $G_{2}(C)$ of $G$ determines $G$-bordism.
\end{abstract}

1. Preliminaries. Throughout the note we will take $G$ to be a finite group. By a $G$-manifold we will mean a differential compact manifold with a differential action of $G$ on it. A family $\mathscr{F}$ in $G$ is a collection of subgroups of $G$ such that if $H \in \mathscr{F}$, then all the subgroups of $H$ and all the conjugates of $H$ are in $\mathscr{F}$. Let $\mathscr{F}^{\prime} \subset \mathscr{F}$ be families in $G$ such that $\exists$ a central element $a$ in $G$ of order 2 such that

(i) $a \notin H, \forall H \in \mathscr{F}-\mathscr{F}^{\prime}$

(ii) $H \in \mathscr{F}^{\prime} \Rightarrow[H \cup\{a\}] \in \mathscr{F}^{\prime}$

(iii) The intersection $S$ of all members of $\mathscr{F}-\mathscr{F}^{\prime}$ is in $\mathscr{F}-\mathscr{F}^{\prime}$. We call such a pair $\left(\mathscr{F}, \mathscr{F}^{\prime}\right)$ of families an admissible pair of families in $G$ with respect to $a \in G$.

EXAMPLE 2.1. Let $G$ be a finite group. We can write the 2-central component $G_{2}(C)$ as $\mathbf{Z}_{2}^{r}=\left[t_{1}, \ldots, t_{r}\right]$, where $t_{1}, \ldots, t_{r}$ are generators of $\mathbf{Z}_{2}^{r}$ with $t_{i}^{2}=$ the identity element and $t_{i} t_{j}=t_{j} t_{i}$. Let $\mathscr{F}_{k}$ be the family of all subgroups of $G$ not containing $\mathbf{Z}_{2}^{k}, 0<k \leq r$, where $\mathbf{Z}_{2}^{k}$ denotes the subgroup of $G$ generated by the first $k$ generators $t_{1}, \ldots, t_{k}$. Then $\left(\mathscr{F}_{k+1}, \mathscr{F}_{k}\right)$ is an admissible pair with respect to $t_{k+1}, 0<k<r$.

2. Stationary point free action of $G_{2}(C)$ and $G$-bordism. The object of this section is to show that if $(M, \theta)$ is a $G$-manifold with the stationary point free action of $G_{2}(C)$ then $(M, \theta)$ is $G$-boundary. Following the notation of Stong [2], let $\mathfrak{N}_{*}\left(G ; \mathscr{F}, \mathscr{F}^{\prime}\right)$ denote the $\left(\mathscr{F}, \mathscr{F}^{\prime}\right)$-free $G$ bordism group for a pair $\left(\mathscr{F}, \mathscr{F}^{\prime}\right)$ of families in $G$. For a given family $\mathscr{F}$ 
in $G$ and an element $g$ in $G$, let $\mathscr{F}_{g}$ denote the smallest family in $G$ consisting of all subgroups $[H \cup\{g\}], H \in \mathscr{F}$.

THEOREM 3.1. If $\left(\mathscr{F}, \mathscr{F}^{\prime}\right)$ is an admissible pair of families in $G$ with respect to a in $G$, then an $\left(\mathscr{F}, \mathscr{F}^{\prime}\right)$-free element in $\mathfrak{R}_{*}\left(G, \mathscr{F}, \mathscr{F}^{\prime}\right)$ is zero in $\mathfrak{N}_{*}\left(G ; \mathscr{F}_{a}, \mathscr{F}_{a}^{\prime}\right)$.

Proof. Let $[M, \theta]$ be in $\mathfrak{R}_{*}\left(G, \mathscr{F}, \mathscr{F}^{\prime}\right)$. Let $F$ denote the fixed points set of $S$ in $M, S$ being the intersection of all the members of $\mathscr{F}-\mathscr{F}^{\prime}$. Since $\mathscr{F}-\mathscr{F}^{\prime}$ is invariant under conjugation, $S$ is normal in $G$ and hence the action $\theta$ on $M$ induces an action on $F$ which we denote once again by $\theta$. Let $\nu$ be the normal bundle of the imbedding of $F$ in the interior of $M$ and $D(\nu)$ be its disc bundle with the action $\theta^{*}$ of $G$ on $D(\nu)$ induced by the real vector bundle maps covering the action $\theta$ on $F$. Since $F$ is fixed point set of $S, a \notin H, \forall H \in \mathscr{F}-\mathscr{F}^{\prime}$ and no point of $F$ is fixed by the subgroup $[S \cup\{a\}]$ generated by $S \cup\{a\}, a$ will act freely on $F$ and hence on $D(\nu)$. Let $F^{\prime}=F /[a]$ and $D^{\prime}(\nu)=D(\nu) /[a]$. Since $a$ is central the actions $\theta$ and $\theta^{*}$ on $F$ and $D(\nu)$ induce actions $\theta^{\prime}$ and $\theta^{* \prime}$ on $F^{\prime}$ and $D^{\prime}(\nu)$ respectively. Let $C_{1}$ and $C_{2}$ be the mapping cylinders of the equivariant double covers $q_{1}: F \rightarrow F^{\prime}$ and $q_{2}: D(\nu) \rightarrow D^{\prime}(\nu)$ respectively and $\psi_{1}$ and $\psi_{2}$ be the induced actions on $C_{1}$ and $C_{2}$ respectively. We have the following commutative diagram

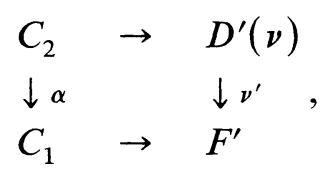

where $\alpha: C_{2} \rightarrow C_{1}$ is the map induced from $\nu^{\prime}: D^{\prime}(\nu) \rightarrow F^{\prime}$ by going to mapping cylinders. Clearly $\partial C_{1}$ is homeomorphic to $F, \alpha^{-1}\left(\partial C_{1}\right)$ is homeomorphic to $D(\nu)$ and the action $\psi_{1}$ on $\alpha^{-1}\left(\partial C_{1}\right)$ is isomorphic to the action $\theta^{*}$ on $D(\nu)$. Consider

$$
W=(M \times[0,1]) \cup C_{2} / \sim,
$$

where $\sim$ is the equivalence relation in $W$ obtained by identifying $D(\nu) \times$ $\{1\}$ with $\alpha^{-1}\left(\partial C_{1}\right)$. Let the action $\phi$ of $G$ on $W$ be given by $\phi \mid M \times[0,1]$ $=\theta \times 1$ and $\phi \mid C_{2}=\psi_{1}$. Take $V$ to be $(\partial M \times[0,1]) \cup(M \times\{1\}-$ $\left.(D(\nu) \times\{1\})^{\circ}\right) \cup\left(\partial C_{2}-\left(\alpha^{-1}\left(\partial C_{1}\right)\right)^{\circ}\right)$, where ${ }^{\circ}$ denotes the interior operator. Since $S$ is the intersection of all the members of $\mathscr{F}-\mathscr{F}^{\prime}, V$ will be $\left(\mathscr{F}_{a}{ }^{\prime}, \mathscr{F}_{a}{ }^{\prime}\right)$-free. Also $W$ is $\left(\mathscr{F}_{a}^{\prime}, \mathscr{F}_{a}^{\prime}\right)$-free and $\partial W$ is homeomorphic to $M \cup V$ by identifying $\partial V$ with $\partial M$. This shows that $[M, \theta]$ is zero in $\mathfrak{R}_{*}\left(G ; \mathscr{F}_{a}, \mathscr{F}_{a}^{\prime}\right)$. 
Let $\mathfrak{A}$ denote the family of all subgroups of $G$ and $\mathscr{F}_{0}$ denote the empty family. Then following the notations of Example 2.1 and using the above Theorem, one immediately gets the following.

Corollary 3.2. For every $k, 0 \leq k<r$, the homomorphism $\mathfrak{N}_{*}\left(G ; \mathscr{F}_{k+1}, \mathscr{F}_{k}\right) \rightarrow \mathfrak{N}_{*}\left(G ; \mathfrak{A}, \mathscr{F}_{k}\right)$ induced from the inclusion map $\left(\mathscr{F}_{k+1}, \mathscr{F}_{k}\right) \rightarrow\left(\mathfrak{A}, \mathscr{F}_{k}\right)$ is zero.

Proof. Since $\left(\mathscr{F}_{k+1}, \mathscr{F}_{k}\right)$ is admissible pair of families with respect to $t_{k+1}$ for $0 \leq k<r$ and no point of the submanifold $V$ in the above construction is fixed by $\mathbf{Z}_{2}^{k}$. Theorem 3.1 gives the Corollary immediately.

COROllary 3.3. Let $\mathbf{P}$ be the family of all subgroups of $G$ which do not contain $G_{2}(C)$. Then the homomorphism $\mathfrak{N}_{*}(G ; \mathbf{P}) \rightarrow \mathfrak{N}_{*}(G ; \mathfrak{A})$ induced from the inclusion map $\mathbf{P} \rightarrow \mathfrak{A}$ is the zero homomorphism.

Proof. By Corollary 3.2, one gets that

$$
\mathfrak{R}_{*}\left(G ; \mathscr{F}_{k+1}, \mathscr{F}_{k}\right) \stackrel{i_{*}}{\rightarrow} \mathfrak{N}_{*}\left(G ; \mathfrak{A}, \mathscr{F}_{k}\right)
$$

is the zero homomorphism, $0 \leq k<r$. Consider the exact bordism sequence for the triple

$$
\begin{aligned}
\left(\mathfrak{A}, \mathscr{F}_{k+1}, \mathscr{F}_{k}\right) & \rightarrow \cdots \mathfrak{R}_{*}\left(G ; \mathscr{F}_{k+1}, \mathscr{F}_{k}\right) \stackrel{i_{*}}{\rightarrow} \mathfrak{N}_{*}\left(G ; \mathfrak{A}, \mathscr{F}_{k}\right) \\
& \stackrel{i_{*}}{\rightarrow} \mathfrak{N}_{*}\left(G ; \mathfrak{U}, \mathscr{F}_{k+1}\right) \rightarrow \cdots
\end{aligned}
$$

where $j_{*}$ is the homomorphism induced from the inclusion $j:\left(\mathfrak{A}, \mathscr{F}_{k}\right) \rightarrow$ $\left(\mathfrak{A}, \mathscr{F}_{k+1}\right.$ ). Since $i_{*}$ is the zero homomorphism, $j_{*}$ will be a monomorphism. Therefore the composite

$$
\mathfrak{N}_{*}\left(G ; \mathfrak{A}, \mathscr{F}_{o}\right) \rightarrow \mathfrak{N}_{*}\left(G ; \mathfrak{A}, \mathscr{F}_{1}\right) \rightarrow \cdots \rightarrow \mathfrak{N}_{*}\left(G ; \mathfrak{A}, \mathscr{F}_{r}\right)
$$

is a monomorphism and hence by the exact bordism sequence of the triple $\left(\mathfrak{A}, \mathscr{F}_{r}, \mathscr{F}_{0}\right)$, one get that $\mathfrak{N}_{*}\left(G ; \mathscr{F}_{r}, \mathscr{F}_{0}\right) \rightarrow \mathfrak{N}_{*}\left(G ; \mathfrak{A}, \mathscr{F}_{0}\right)$ is the zero homomorphism. This completes the proof since $\mathscr{F}_{r}=\mathbf{P}$ and $\mathscr{F}_{0}=\varnothing$.

COROLlaRY 3.4. If $G_{2}(C)$ acts on $M$ under $\theta$ without any stationary point then $(M, \theta)$ is a G-boundary.

3. The stationary points set $F_{G_{2}(C)}$ and the normal bundle. In the last section we dealt with the case when $F_{G_{2}(C)}$ is empty. In this section we consider the case when $F_{G_{2}(C)} \neq \varnothing$. For this we introduce the concept of 
equivariant trivial normal bundle and use this concept to settle the case $F_{G_{2}(C)} \neq \varnothing$ in the form of Theorem 4.2.

Let $\left(M^{n}, \theta\right)$ be a closed $G$-manifold. Consider the decomposition of $F=F_{G_{2}(C)}\left(M^{n}\right)$ as $F=\bigcup_{l=0}^{n} F^{l}$, where $F^{l}$ denotes the $l$-dimensional component of $F$. Let $\mathscr{D}\left(\nu_{l}\right)$ be the normal disc bundle of $F^{l}$ in $M^{n}$ with the induced action $\theta_{l}$ of $G$ on $\mathscr{D}\left(\nu_{l}\right)$.

DEFINITION 4.1. $F$ is said to have an equivariant trivial normal bundle in $M^{n}$, if $G / G_{2}(C)$ acts trivially on $F$ and $\exists$ some positive dimensional $G$-representations $\left(W_{l}, \phi_{l}\right), 0 \leq l \leq n$, such that in $\mathfrak{N}_{*}(G ; \mathfrak{A}, \mathbf{P})$

$$
\left[D\left(\nu_{l}\right), \theta_{l}\right]=\left[F^{l}\right]\left[D\left(W_{l}\right), \phi_{l}\right] \text {, }
$$

$D\left(W_{l}\right)$ being the unit disc of $W_{l}$.

Let $\left\{V_{k}, \psi_{k}\right\}_{1 \leq k \leq m}$ be the finite set of all irreducible representations of $G$. Let $\mathbf{Z}^{+}$be the set of all non-negative integers. Then any $G$-representation can be written as $(V(f), \psi(f))$ for some map $f:\{1, \ldots, m\} \rightarrow \mathbf{Z}^{+}$ where $V(f)=\oplus_{k=1}^{m}\left(V_{k}, \psi_{k}\right)^{f(k)},\left(V_{k}, \psi_{k}\right)^{f(k)}$ being the direct sum of $f(k)$ copies of $\left(V_{k}, \psi_{k}\right)$. Let us denote the unit disc and the unit sphere of $V(f)$ by $D(f)$ and $S(f)$.

THEOREM 4.2. If $F$ has an equivariant trivial normal bundle in $M^{n}$, then $F$ is a boundary and $\left(M^{n}, \theta\right)$ is a G-boundary.

Proof. Since $F$ has an equivariant trivial normal bundle in $M^{n}$, we have

$$
\left[\mathscr{D}\left(\nu_{l}\right), \theta_{l}\right]=\left[F^{l}\right]\left[D\left(W_{l}\right), \theta_{l}\right]
$$

for some positive dimensional $G$-representations $\left(W_{l}, \phi_{l}\right), 0 \leq l \leq n$. Also $\left(W_{l}, \phi_{l}\right)=\left(V\left(f_{l}\right), \psi\left(f_{l}\right)\right)$ for some map $f_{l}:\{1, \ldots, m\} \rightarrow \mathbf{Z}^{+}$. Therefore

$$
\left[\mathscr{D}\left(\nu_{l}\right), \theta_{l}\right]=\left[F^{l}\right]\left[D\left(f_{l}\right), \psi\left(f_{l}\right)\right] \text {. }
$$

Let $i_{*}: \mathfrak{N}_{*}(G ; \mathfrak{A}) \rightarrow \mathfrak{N}_{*}(G ; \mathfrak{A}, \mathbf{P})$ be the homomorphism induced by the inclusion map $i:(\mathfrak{A}, \phi) \rightarrow(\mathfrak{A}, \mathbf{P})$. Then

$$
i_{*}\left[M^{n}, \theta\right]=\sum_{l=0}^{n}\left[\mathscr{D}\left(\nu_{l}\right), \theta_{l}\right]=\sum_{l=0}^{n}\left[F^{l}\right]\left[D\left(f_{l}\right), \psi\left(f_{l}\right)\right] .
$$

Therefore

$$
\partial_{*} i_{*}\left[M^{n}, \theta\right]=\sum_{l=0}^{n}\left[F^{l}\right]\left[S\left(f_{l}\right), \psi\left(f_{l}\right)\right]=0
$$


in $\mathfrak{N}_{*}(G ; \mathbf{P})$, where $\partial_{*}: \mathfrak{R}_{*}(G ; \mathfrak{A}, \mathbf{P}) \rightarrow \mathfrak{R}_{*}(G ; \mathbf{P})$ is the boundary homomorphism. Therefore $\exists$ a P-free $G$-manifold $(D, \eta)$ such that

$$
(\partial D, \eta)=\bigcup_{l=0}^{n}\left(F^{l} \times\left(S\left(f_{l}\right), \psi\left(f_{l}\right)\right)\right) .
$$

Since $\left(W_{l}, \phi_{l}\right)$ is positive dimensional $G$-representation, $\forall l, \exists$ a member $k(l)$ in the set $\{1, \ldots, m\}$ such that $f_{l}(k(l)) \neq 0$. Consider the irreducible $G$-representation $\left(V_{k(l)}, \psi_{k(l)}\right)$. Let $\left(\tilde{V}_{k(l)}, \tilde{\psi}_{k(l)}\right)$ be an irreducible component of the $G_{2}(C)$-representation induced by the $G$-representation $\left(V_{k(l)}, \psi_{k(l)}\right)$. Then $\exists$ a subgroup $H_{k(l)}$ of $G$ isomorphic to $\mathbf{Z}_{2}^{r-1}$ which fixes $\tilde{V}_{k(l)}, G_{2}(C)$ being $\mathbf{Z}_{2}^{r}$. Let us fix some $\beta, 0 \leq \beta \leq n$.

From the equation (1), we get

$$
F_{H_{k(\beta)}}(\partial D, \eta)=F_{H_{k(\beta)}}\left(\bigcup_{l=0}^{n}\left(F^{l} \times\left(S\left(f_{l}\right), \psi\left(f_{l}\right)\right)\right)\right) .
$$

Let $F_{H_{k(\beta)}}(D)=F^{*}$ and $\mathbf{Z}_{2, \beta} \approx \mathbf{Z}_{2}$ be the complement of $H_{k(\beta)}$ in $G_{2}(C)$ $=\mathbf{Z}_{2}^{r}$. Then one gets

$$
\left(\partial F^{*}, \eta \mid \mathbf{Z}_{2, \beta}\right)=\bigcup_{l=0}^{n}\left(F^{l} \times\left(S^{\Delta(l, \beta)-1}, a\right)\right),
$$

where $a$ is the antipodal involution and the integer $\Delta(l, \beta)$ is the nonnegative integer depending on $l$ and $\beta$. Since $H_{k(\beta)}$ fixed $\tilde{V}_{k(\beta)}$ and $f_{\beta}(k(\beta)) \neq 0$, one infers that $\Delta(\beta, \beta) \geq 1$. Since $D$ is $\mathbf{P}$-free, $\mathbf{Z}_{2, \beta}$ will act freely on $F^{*}$ and therefore $\left[\partial F^{*}, \eta \mid \mathbf{Z}_{2, \beta}\right]$ is zero in $\mathfrak{N}_{*}\left(\mathbf{Z}_{2, \beta} ; \mathscr{F}_{1}\right), \mathscr{F}_{1}$ being the family consisting of only trivial subgroup of $\mathbf{Z}_{2, \beta}$. This gives

$$
\sum_{l=0}^{n}\left[F^{l}\right]\left[S^{\Delta(l, \beta)-1}, a\right]=0
$$

in $\mathfrak{R}_{*}\left(\mathbf{Z}_{2, \beta} ; \mathscr{F}_{1}\right)$. But $\mathfrak{R}_{*}\left(\mathbf{Z}_{2, \beta} ; \mathscr{F}_{1}\right)$ is free $\mathfrak{N}_{*}$-module with a set $\left\{\left[S^{n}, a\right], n \in \mathbf{Z}^{+}\right\}$of generators. This together with the fact that $\Delta(\beta, \beta)$ $\geq 1$ gives $\left[F^{\beta}\right]=0$ in $\Re_{*}$. By varying $\beta$, one gets $\left[F^{\beta}\right]=0, \forall \beta=0, \ldots, n$. Hence $[F]=0$ in $\mathfrak{N}_{*}$. Therefore

$$
i_{*}\left[M^{n}, \theta\right]=\sum_{l=0}^{n}\left[F^{l}\right]\left[D\left(f_{l}\right), \psi\left(f_{l}\right)\right]=0 \quad \text { in } \mathfrak{A}_{*}(G ; \mathfrak{A}, \mathbf{P}) .
$$

But from Corollary 3.3, one infers that $i_{*}: \mathfrak{R}_{*}(G, \mathfrak{A}) \rightarrow \mathfrak{R}_{*}(G ; \mathfrak{A}, \mathbf{P})$ is an injection. Therefore $\left[M^{n}, \theta\right]$ is zero in $\mathfrak{N}_{*}(G ; \mathfrak{U})$. 


\section{S. S. KHARE}

\section{REFERENCES}

[1] P. E. Conner, and E. E. Floyd, Differentiable periodic maps, Springer-Verlag, Berlin, 1964.

[2] R. E. Stong, Equivariant bordism and $\left(\mathbf{Z}_{2}\right)^{k}$ actions, Duke Math. J. 371970.

Received June 17, 1983 and in revised form July 29, 1983. The present work was partially supported by D. A. E. Grant.

DePartment of Mathematics

NORTH-EASTERN HILL UNIVERSITY

BiJNi CAMPUS, SHILlong 793003

Meghalaya, India 


\section{PACIFIC JOURNAL OF MATHEMATICS \\ EDITORS}

Donald BABBITT (Managing Editor)

University of California

Los Angeles, CA 90024

J. Dugundu

University of Southern Californa

Los Angeles, CA 90089-1113

R. FINN

Stanford University

Stanford, CA 94305

HermanN FLaSChKa

University of Arizona

Tucson, AZ 85721

\author{
C. C. MOORE \\ University of California \\ Berkeley, CA 94720 \\ ARTHur Ogus \\ University of California \\ Berkeley, CA 94720 \\ Hugo Rossi \\ University of Utah \\ Salt Lake City, UT 84112 \\ H. SAMELSON \\ Stanford University \\ Stanford, CA 94305
}

ASSOCIATE EDITORS
R. ARENS
E. F. BECKENBACH
B. H. NeUmanN
F. WOLF
K. YOSHIDA (1906-1982)

\section{SUPPORTING INSTITUTIONS}

UNIVERSITY OF ARIZONA

UNIVERSITY OF BRITISH COLUMBIA

UNIVERSITY OF OREGON

CALIFORNIA INSTITUTE OF TECHNOLOGY

UNIVERSITY OF CALIFORNIA

MONTANA STATE UNIVERSITY

UNIVERSITY OF SOUTHERN CALIFORNIA

UNIVERSITY OF NEVADA, RENO

STANFORD UNIVERSITY

UNIVERSITY OF HAWAII

NEW MEXICO STATE UNIVERSITY

UNIVERSITY OF TOKYO

UNIVERSITY OF UTAH

WASHINGTON STATE UNIVERSITY

OREGON STATE UNIVERSITY

UNIVERSITY OF WASHINGTON 


\section{Pacific Journal of Mathematics}

\section{Vol. 116, No. $1 \quad$ November, 1985}

K. Adachi, Le problème de Lévi pour les fibrés grassmanniens et les variétés

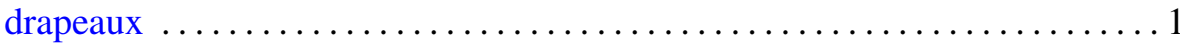

John MacLeod Ball, Remarks on the paper: "Basic calculus of variations" . . . 7 John Kelly Beem and Phillip E. Parker, Whitney stability of solvability . . . 11 Alberto Facchini, Decompositions of algebraically compact modules .......25

S. S. Khare, Finite group action and equivariant bordism $\ldots \ldots \ldots \ldots . \ldots 39$

Horst Leptin, A new kind of eigenfunction expansions on groups $\ldots \ldots \ldots . .45$

Pei-Kee Lin, Unconditional bases and fixed points of nonexpansive

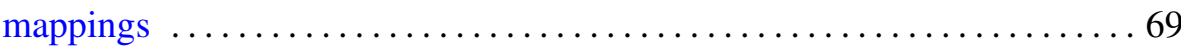

Charles Livingston, Stably irreducible surfaces in $S^{4} \ldots \ldots \ldots \ldots \ldots 77$

Kevin Mor McCrimmon, Nonassociative algebras with scalar involution . . .885

Albert Milani, Singular limits of quasilinear hyperbolic systems in a

bounded domain of $\mathbf{R}^{3}$ with applications to Maxwell's equations

Takemi Mizokami, On $M$-structures and strongly regularly stratifiable

spaces

Jesper M. Møller, On the homology of spaces of sections of complex

projective bundles

Nikolaos S. Papageorgiou, Carathéodory convex integrand operators and

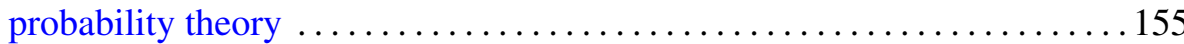

Robert John Piacenza, Transfer in generalized prestack cohomology 185

Lance W. Small and Adrian R. Wadsworth, Integrality of subrings of matrix rings ...

James Michael Wilson, On the atomic decomposition for Hardy spaces 